Revista de Comunicación y Salud, 2018, Vol. 8, no 1, pp. 61-70

Editado por Cátedra de Comunicación y Salud

ISSN: 2173-1675

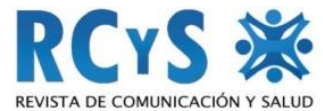

Enviado 17 de mayo de 2018

Aprobado 28 de junio de 2018

\title{
VALIDACIÓN DE LA ESCALA DE PERCEPCIÓN DE INCLUSIÓN DOCENTE DE LA NIÑEZ MIGRANTE (PID)
}

\author{
Validation of perception scale of teacher inclusion of migrant children (PID) \\ Alicia Monzalvo Curiel ${ }^{1}$ Universidad de Sonora. México. \\ Daniel Rojas Méndez \\ Nissa Yaing Torres Soto
}

Agradecimientos: Al Dr. Antonio Medina Rivilla de la Universidad Nacional de Educación a Distancia de España y al Dr. Juan Carlos Manríquez de la Universidad de Sonora por su valiosa colaboración.

\section{Resumen}

Los estudiantes migrantes comparten algunos aspectos en común, por ejemplo los efectos psicosociales que implica llegar a un nuevo contexto escolar. Todos los niños y en especial los migrantes, tienen la necesidad de ser atendidos en programas que contemplen el apoyo para su regularización y atención a sus necesidades educativas especiales. El objetivo de este artículo es validar la escala de Percepción de Inclusión Docente (PID), que se diseñó para ser empleada en poblaciones migrantes de nivel básico (Monzalvo, en prensa).Se incluye un análisis factorial confirmatorio y el cálculo de la confiabilidad de la escala, la cual fue confiable $(\alpha=.90)$. La escala resultante está compuesta por 26 ítems agrupados en tres factores. Para la validación se aplicó el cuestionario a niños migrantes y regulares del segundo y tercer ciclo (primaria alta) en escuelas públicas y privadas comprendiendo planteles de la ciudad de Hermosillo; incluyendo el Poblado Miguel Alemán y Ejidos ubicados en la carretera Hermosillo-Bahía Kino en el estado de Sonora, México $(n=328)$. Nuestros resultados contribuyen a una mejor comprensión de las necesidades de inclusión de los niños migrantes, así como para una revisión futura de políticas públicas para el logro de programas puente o de protocolos de inserción de estudiantes migrantes al sistema educativo mexicano.

Palabras clave: inclusión, niñez migrante, competencia socio emocional.

\begin{abstract}
Migrant students share some aspects and the migration process affects them in a variety of ways, for example psychosocial effects when reaching a new school context. All children, and especially migrants, need to be included in programs that provide the support they need for academic regularization and attention to their special educational needs. The objective of this article is to validate the Teacher Inclusion Perception Scale (PID) that was design for elementary school level with migrant populations (Monzalvo, in press). It includes a confirmatory factor analysis and the calculation of the reliability of the scale, which was reliable $(\alpha=.90)$. The resulting scale includes 26 items grouped into three factors. For the validation, the questionnaire was applied to migrant and regular children of the second and third cycle (upper elementary school) in public and private schools, including those in the city of Hermosillo, that comprehends Miguel Aleman and communal lands located in Hermosillo-Bahía Kino highway in the state of Sonora, Mexico $(n=328)$. Our results contribute to a better understanding of the inclusion needs of migrant children, as well as to a future review of public policies to achieve a protocol for the integration of migrant students into the Mexican educational system.
\end{abstract}

Keywords: inclusion, migrant children, socioemotional competence.

${ }^{1}$ Autor para correspondencia: Alicia Monzalvo Curiel. Dra. en Ciencias Sociales. aliciamonzalvo@yahoo.com.mx 
Validación de la escala de percepción de inclusión docente de la niñez migrante (PID)

\begin{tabular}{|l|}
\hline Cómo citar el artículo \\
Monzalvo Curiel, A., Rojas Méndez, D., y Torres Soto, N. Y. (2018). Validación de la escala de \\
percepción de inclusión docente de la niñez migrante (PID). Revista de Comunicación y Salud, \\
8(1), pp. 61-70. DOI: http://doi.org/10.35669/revistadecomunicacionysalud.2018.8(1).61-70
\end{tabular}

\section{Introducción}

La niñez migrante enfrenta diferentes retos y obstáculos para su ingreso a los planteles educativos, sin embargo, en el sistema educativo mexicano, actualmente no se considera ningún tipo de protocolo para la inclusión educativa de niños migrantes. A pesar del importante rol del docente para la educación inclusiva, no necesariamente se le brindan las herramientas necesarias para atender a los estudiantes migrantes, por ejemplo con cursos de sensibilización intercultural o competencias socioemocionales (CSE) que pudieran ayudarlo en el desarrollo de la empatía para el trabajo con grupos minoritarios y en situación vulnerable. La (CSE) es un factor importante que contribuye para crear un contexto de inclusión en el aula. Se ha estudiado y discutido mucho en los últimos años la importancia de contar con planes de estudio que preparen de manera efectiva a los profesores en formación para una educación inclusiva (San Martin et al., 2017). La revisión de la literatura muestra que no hay un consenso respecto de qué se entiende por Competencia Socio Emocional (CSE), sin embargo, algunos modelos teóricos consideran a la empatía como componente del constructo de competencia socioemocional (Bisquerra, 2003; Bisquerra, et al., 2007) es decir, el reconocer lo que los demás están sintiendo, lo que es a su vez un componente clave para el aula inclusiva. Se considera que si el docente puede entender al estudiante y adaptarse a sus diferencias y necesidades, le ofrece un espacio único en el que su estudiante lo pueda percibir como inclusivo.

La educación inclusiva intenta comprender una gran cantidad de grupos a los que históricamente se les ha negado la posibilidad de participar con equidad en los mismos espacios que la mayoría de la población, por ejemplo, personas de origen indígena, extranjeros o mujeres (Cruz, 2007). Lamentablemente, existe ausencia de información para la ubicación y seguimiento de la trayectoria migratoria de la niñez migrante en el estado de Sonora. En ocasiones, los menores sufren rezago social, lo que imposibilita la atención merecida. Los grupos de migrantes identificados para este estudio, son los nacionales que migran de una ciudad a otra y hablan español, los de origen indígena que tienen un idioma nativo distinto al de su plantel educativo, y los migrantes externos de origen mexicano o extranjero que han llegado al estado de Sonora en los últimos dos años.

Las necesidades educativas especiales de los educandos y las instancias de apoyo como las Unidades de Servicio de Apoyo a la Educación Regular (USAER), son las instancias técnicas operativas de apoyo a la atención de estudiantes con necesidades educativas especiales y/o discapacidad integradas en escuelas de educación básica (SEP, 2006). Estos servicios, se limitan principalmente al apoyo de estudiantes con discapacidad o problemas de tipo conductual. De acuerdo al informe de UNESCO (2015), contar con un programa para la intervención temprana con estudiantes migrantes y/o refugiados de los países involucrados, permitiría impulsar el crecimiento económico del país. De ahí la importancia de contar con instrumentos que permitan medir las necesidades de los niños migrantes de sentirse incluidos por parte del personal docente y de la institución educativa receptora que los alberga.

Según el Instituto Nacional para la Evaluación de la Educación (INEE, 2017), algunos maestros y autoridades educativas muestran actitudes discriminatorias hacia los miembros de las comunidades indígenas. Cabe destacar que, a los docentes, no necesariamente se les capacita ni apoya con materiales que puedan orientarlo hacia una sensibilidad intercultural y con eso ayudar al proceso de adaptación al aula del niño migrante. Dado el difícil proceso por el que pasan los menores con barreras de idioma, separación familiar y nuevos entornos, es primordial la atención emocional para ayudarle en su proceso de adaptación. Al respecto, Bisquerra (2001) afirma que la educación emocional es un proceso educativo esencial para potenciar el desarrollo emocional y cognitivo del niño. Por lo tanto, es importante trabajar en los aspectos emocionales e incluir programas de educación emocional en los protocolos de inserción escolar en las escuelas tanto para niños regulares, como migrantes; sin embargo, los sistemas educativos tradicionales, no necesariamente consideran las necesidades emotivas y académicas para los niños en situación migratoria en México. El informe de la Organización de las Naciones Unidas para la Educación, la Ciencia y la 


\section{Validación de la escala de percepción de inclusión docente de la niñez migrante}

(PID)

Cultura (UNESCO) del año 2015, propone contar con programas que permitan a la población estudiantil migrante regularizarse académicamente, así como preparar al docente para poder atender a la diversidad de estudiantes que ingresan al sistema educativo. Ya son muchos los países que se suman a contar con protocolos para la atención a niños migrantes, por ejemplo Finlandia, país Europeo que, a pesar de su poca población, ha destacado por sus resultados en el Programa Internacional para la Evaluación de Estudiantes (PISA) en las últimas décadas. En contraste, de acuerdo a informes de la Organización para la Cooperación y el Desarrollo Económico (OCDE, 2018), los estudiantes mexicanos obtienen menores puntajes en matemáticas que otros países y muestran un claro retraso de hasta dos años comparado con sus pares.

En el artículo $3^{\circ}$ de la Constitución Mexicana se establece como principios de la educación en México, la gratuidad, la laicidad y la calidad, pero para lograrlo hay retos y desafíos que vencer. Cada vez emigran más personas e incrementan las necesidades para atender a la población repatriada en México. Un escaso valor o incluso rechazo a su experiencia migratoria y de sus habilidades adquiridas, pueden inhibir la adaptación del migrante (Mojica, 2017). Es así que el país se encuentra inmerso en el complejo problema migratorio y adicionalmente, las escuelas requieren planeación y adaptación a las necesidades de los niños migrantes. Si bien, las transformaciones en las políticas públicas han permitido la educación de niños migrantes en las aulas en México sin tanta restricción de documentos, esto no ha sido suficiente para darle atención a sus necesidades específicas de nivelación de académica y de idioma, así como las emocionales.

Se conoce poco sobre la percepción que tienen los niños migrantes en cuanto a la atención que reciben en los centros escolares a su llegada. ¿Cómo se da el seguimiento a su trayectoria migratoria? ¿Qué experimenta el niño migrante en los planteles mexicanos? Pero sobre todo, ¿cuáles son las adaptaciones que su docente realiza para incluirlo en el aula? Si bien, poco se explora acerca de las vivencias de la niñez migrante, este estudio permite analizar las percepciones de los niños en cuanto a su institución, docente y compañeros de clase.

Estudios recientes han identificado algunos factores que son claves en la dinámica de la inclusión en las aulas, tales como el hecho de contar con recursos adecuados, el plan de estudios, los conocimientos que se transmiten, las creencias, las actitudes y las emociones que experimentan los docentes (Domović et al., 2017).

Es evidente que los docentes constituyen uno de los pilares principales para que se fomente la inclusión y se atienda la diversidad de necesidades educativas de los estudiantes en el aula (Arnaiz, 2003). Numerosos estudios han puesto en evidencia, que la percepción que tienen los docentes sobre la educación inclusiva puede verse influenciada por los antecedentes del docente y las características particulares de los estudiantes (Hsieh et al., 2012) (Tejada et al., 2012). También se ha reportado que los docentes pueden experimentar algunas emociones negativas como miedo, frustraciones, enojo y poca preparación al momento de trabajar bajo el modelo de clase inclusiva, por el hecho de creer que pueden conducir a bajos estándares académicos (Chiner y Cardona, 2013; D'Haem y Griswold, 2017; Gao y Mager, 2011). Inclusive, algunos investigadores han evidenciado que los cambios en las legislaciones y las políticas en materia de inclusión no han tomado en cuenta las emociones que experimentan los docentes como el estrés percibido al momento de conducir una educación inclusiva (Forlin et al., 2014; Rowan et al., 2017). De ahí la importancia de contar con programas continuos de capacitación docente para orientarlo a tener aulas inclusivas.

En este sentido, la inclusión educativa por parte de los docentes está asociada con factores externos que aluden a la implementación de políticas públicas de inclusión educativa, pero también, con factores internos como las actitudes de disposición y aceptación del docente durante su quehacer en el aula (Young et al., 2017). Al respecto, Espinoza (2006) sostiene que el rendimiento académico de los estudiantes está determinado por variables psicológicas como la autoconfianza y la autoestima.

En este sentido, es razonable pensar que si el trato del docente hacia sus estudiantes no es favorable, los resultados académicos de los estudiantes se verán igualmente afectados al no sentirse valorados por el profesor. En cambio, si la actitud del docente hacia sus estudiantes es adecuada, el proceso formativo será exitoso (Amat, 1998).

Por otra parte, existe evidencia empírica que algunas medidas que podrían beneficiar a los docentes para crear un ambiente escolar optimo e inclusivo es a través del fortalecimiento de la calidad docente, incluyendo el aumento de inversión presupuestal en materia de inclusión y el hecho

Revista de Comunicación y Salud, 2018, Vol. 8, nº 1, pp. 61-70 
de proporcionar espacios adecuados para la enseñanza de los estudiantes (Li, Zou y Wang, 2009; Tangen y Beutel, 2017).

En el contexto mexicano, los docentes generalmente están centrados en la enseñanzaaprendizaje de sus estudiantes y se desvincula el componente emotivo dentro de la dinámica escolar. Por esta razón, se requiere de mayor investigación sobre la Percepción de la Inclusión Docente en la niñez migrante.

Analizando la desarticulación entre la política pública y las prácticas educativas en México, representa un reto resolver las necesidades educativas de las poblaciones migrantes. Es decir, en México aún no se han logrado enseñanzas que puedan agregarse al currículo del docente de acuerdo a la situación que se vive en el país, desde este contexto de inclusión educativa.

El término inclusión ha sido muy utilizado en las últimas décadas en México, pero ha sido difícil su implementación para lograr equidad y calidad para todos los estudiantes.

El contexto adverso y la xenofobia en la que se puede encontrar a la población de minoría étnica en Estados Unidos en el gobierno actual del presidente Trump, se ha extendido a diferentes naciones, y puede representar una forma de rechazo a ciertos grupos como formas de intolerancia a lo que los docentes deben estar atentos, no solo con estudiantes repatriados, también con los migrantes indígenas que pueden sufrir discriminación.

En México, existe una gran gama de diversidad estudiantil, como poblaciones indígenas, aproximadamente el $6.5 \%$ de la población habla alguna lengua indígena según cifras del Instituto Nacional de Estadística (INEGI, 2018). Por otro lado, el censo indica que el $6.5 \%$ de la población estudiantil mexicana menor de 19 años ha tenido alguna experiencia educativa internacional. El $74.4 \%$ de las personas que nacieron en otro país y radican en México provienen de Estados Unidos, $3 \%$ de Guatemala, $2.6 \%$ de Colombia y $2.3 \%$ de Venezuela. Entre agosto de 2013 y septiembre de 2018, poco más de 760 mil personas emigraron del país; casi 40 mil personas más que en el periodo 2009 - 2014 (719 242 personas) y setenta de cada cien emigrantes internacionales son hombres.

Sin embargo, existe una ausencia de datos sistematizados por parte de las instituciones responsables para conocer el número de migrantes en las aulas. No se registran a todos los migrantes, ya sea por su lugar de nacimiento, por su estatus migratorio o por la falta de procesos eficientes entre las instituciones involucradas responsables de la recolección de información como el Sistema de Información, Control y Registro Escolar de Sonora (SICRES).

\section{Método}

\subsection{Participantes}

El tipo de muestreo para este trabajo fue por conveniencia, esto de acuerdo a la información recibida en las siguientes instancias públicas:

a) Coordinación Estatal del Programa para la Inclusión y Equidad educativa de la Secretaría de Educación y Cultura del Estado de Sonora (SEC): para ubicación de menores hijos de jornaleros migrantes.

b) Programa Binacional de Educación Migrante (PROBEM): para ubicación de estudiantes de registro al sistema de educación en el estado Sonora provenientes de Estados Unidos $u$ otros países con o sin nacionalidad mexicana a través del Sistema de Información, Control y Registro Escolar de Sonora (SICRES). Ciclo escolar 2016-2017 y 2017-2018.

c) Secretaría de Educación y Cultura (SEC) y el Instituto Nacional para la Evaluación de la Educación (INEE): para ubicación de planteles con matricula de migrantes indígenas.

Se obtuvo una muestra de 328 niños. El instrumento comprendió 26 reactivos aplicados a estudiantes de primaria alta en situación migratoria y a estudiantes regulares de 20 escuelas públicas y privadas entre 7 y 12 años de edad. El $60 \%$ de los estudiantes se encontraban inscritos en escuelas públicas y $40 \%$ en escuelas privadas al momento del levantamiento de datos en la ciudad de Hermosillo; incluyendo el Poblado Miguel Alemán y Ejidos ubicados en la carretera Hermosillo-Bahía Kino en el estado de Sonora, México. 
Validación de la escala de percepción de inclusión docente de la niñez migrante

(PID)

\subsection{Instrumentos}

Para la medición de la Percepción de Inclusión Docente (PID), se utilizaron 3 factores que evalúan: Relación Maestro-Estudiante, compuesto por 8 ítems; Conciencia Social, compuesta por 9 ítems; y Facilidad Institucional, compuesta por 9 ítems; teniendo un total de 26 variables con un coeficiente de alfa de $a=.90$. El instrumento tiene formato tipo Likert con 6 tipos de respuesta: 1 totalmente en desacuerdo, 2 algo en desacuerdo, 3 poco en desacuerdo, 4 poco de acuerdo, 5 algo de acuerdo y 6 totalmente de acuerdo.

\subsection{Procedimiento}

Se aplicaron cuestionarios a los estudiantes migrantes provenientes de México y otros países como Estados Unidos, Cuba, Finlandia, Honduras, Guatemala, Canadá, menores hijos de jornaleros agrícolas y migrantes provenientes del Sur del país como Triquis, Mixtecos y Zapotecos. Para lo anterior se procedió a visitar a decenas de planteles en diferentes zonas ya que en los registros oficiales no necesariamente se tiene información validada que permita su seguimiento.

La distribución de los cuestionarios fue aplicada a partir del muestreo por conveniencia, en una sola intervención de modo transversal, en un intervalo de tiempo de 6 meses, lo que duró el levantamiento de datos. El tiempo aproximado para cada sesión fue de 45 a 60 minutos. A cada participante se le asignó un código de identificación que coincidía con el código del docente, para identificarlo por su escuela y situación regular y migratoria. Algunas escuelas se visitaron al azar para conocer si tenían niños migrantes, aunque no se incluían en los directorios. La situación migratoria para el caso de niños extranjeros y en situación vulnerable, se manejó como confidencial, por lo que se omiten los nombres de las escuelas por principio de confidencialidad.

El cuestionario se utilizó en español, sin embargo, si era necesario se apoyaba a los niños a la traducción al inglés o lengua indígena, de forma que cada sujeto contestó la versión del cuestionario en su lengua materna. Para medir la consistencia interna de esta escala, se utilizó el alfa de Cronbach.

Análisis de datos:

La aplicación se llevó a cabo cumpliendo con todos los requerimientos éticos. Una vez obtenidos los datos, se procedió a analizarlos a través del Paquete Estadístico para las Ciencias Sociales (SPSS) por sus siglas en inglés. Se llevó a cabo un Análisis Factorial Exploratorio (AFE) por componentes principales y rotación Varimax, donde solo se conservaron los indicadores con un peso factorial mayor a > .30 (Field, 2013). Además, se probó el índice de la prueba de Kaiser-MeyerOlkin (KMO), entre mayor se aproxima al 1, las correlaciones son más compactas y producen resultados confiables; y en cuanto a la prueba de esfericidad de Barlett, este debe resultar significativa $(p<.05)$ (Field, 2013). El número de factores fue evaluado a través del porcentaje de varianza explicada para la totalidad de la escala.

También se realizó el análisis de confiabilidad a través del alfa de Cronbach, considerando aceptable un índice de consistencia interna mayor a .60 (Corral, et al., 2001). Y se llevó a cabo un análisis de correlación de Pearson para las variables resultantes de la escala a fin de comprobar el grado de asociación entre las mismas (Restrepo y González, 2007).

En el siguiente paso se efectuó el Análisis Factorial Confirmatorio (AFC) para probar la validez de la escala. Considerando los indicadores prácticos como el Índice de Ajuste Comparativo (CFI), BentlerBonett de Ajuste Normado (BBNFI) y No-Normado (BBNNFI) iguales o mayores a .90 (Bentler y Bonet, 1980), y el Error de Aproximación Cuadrático Medio (RMSEA) igual o menor a .06 (Hu y Bentler, 1995). Se estimó y probó un modelo estructural que presenta los factores de primer orden (relación maestro-estudiante, conciencia social y facilidad institucional) que conformaron el factor de segundo orden denominado "Percepción de Inclusión Docente".

Por otra parte, se llevaron a cabo reuniones con grupos de niños del segundo y tercer ciclo de primaria con el equipo de investigación, con el fin de verificar el contenido y redacción adecuada del instrumento para su estandarización y normalización. En esa fase se evaluó el tiempo que tardaba cada participante en su llenado, las palabras que no entendían y preguntas de difícil comprensión, para obtener sugerencias para la revisión de la redacción de las preguntas y estructura del cuestionario. 
Validación de la escala de percepción de inclusión docente de la niñez migrante (PID)

\section{Resultados}

\section{Análisis factorial exploratorio}

El AFE mostró una estructura de 3 factores con el método de componentes principales y rotación Varimax sobre los 26 reactivos de la escala total y se obtuvo una varianza total explicada de 43.57\%. La escala arrojó un valor de alfa de Cronbach aceptable de 0.90 considerando 26 reactivos que alcanzaron el peso factorial mayor a .30 (Ver Tabla 4). EI KMO fue de .92 y la prueba de esfericidad de Bartlett obtuvo una Chi cuadrada de 2625.024 (300 gl.) la cual resultó significativa $(\mathrm{p}=.000)($ Ver Tabla 1$)$.

Tabla 1. KMO y prueba de Bartlett

\begin{tabular}{lll} 
Medida de adecuación muestral de Kaiser-Meyer-Olkin. & .923 \\
Prueba de esfericidad de Bartlett & Chi-cuadrado aproximado & 2625.024 \\
& Gl & 300 \\
& Sig. & .000 \\
\hline
\end{tabular}

La varianza explicada de los factores logró alcanzar un porcentaje acumulado del $43.5 \%$, esto contrastado con la teoría de las escalas aplicadas en el estudio (Ver Tabla 2).

Tabla 2. Análisis factorial exploratorio con extracción de tres factores para la escala de percepción de inclusión docente.

\begin{tabular}{|c|c|c|c|c|c|c|}
\hline \multirow[t]{2}{*}{ Componente } & \multicolumn{3}{|c|}{ Factorial exploratorio } & \multicolumn{3}{|c|}{ Factorial con extracción } \\
\hline & Total & $\begin{array}{c}\text { \% de la } \\
\text { varianza }\end{array}$ & $\begin{array}{c}\% \text { de la } \\
\text { varianza } \\
\text { acumulada }\end{array}$ & Total & $\begin{array}{c}\% \text { de la } \\
\text { varianza }\end{array}$ & $\begin{array}{c}\% \text { de la } \\
\text { varianza } \\
\text { acumulada }\end{array}$ \\
\hline 1 & 8.033 & 32.01 & 32.01 & 8.003 & 32.01 & 32.01 \\
\hline 2 & 1.663 & 6.65 & 38.66 & 1.663 & 6.653 & 38.66 \\
\hline 3 & 1.227 & 4.90 & 43.57 & 1.227 & 4.907 & 43.57 \\
\hline
\end{tabular}

$\mathrm{KMO}=.923$. Prueba de esfericidad de Barttlet: $\mathrm{X}^{2}=2625.024, \mathrm{gl}=300, \mathrm{p}=.000$. Varianza total $=43.57 \%$.

Alfa total $=.90, \mathrm{n}=328$

El primer componente representa la "relación maestro-estudiante". Se integra por 8 reactivos que explican el $32.01 \%$ de la varianza total explicada, con pesos factoriales que oscilan entre .58 y .72 . Este factor presenta un alfa de Cronbach de .76 con una media general de $M=4.90(D E=0.90)$. El reactivo con la media más alta fue "Me siento cómodo hablando con mis compañeros del lugar de donde vengo" $(M=5.35, \mathrm{DE}=1.38)$. Por el otro lado, el indicador con puntuación más baja fue "Mi maestro conoce exactamente qué tipo de situaciones escolares me molestan" $(M=3.98, D E=1.73)$. El segundo componente denominado "conciencia social" describe el $6.65 \%$ de la varianza con puntuaciones entre .51 y .68 con una consistencia interna de .75 con una media general de $\mathrm{M}=4.92$ $(\mathrm{DE}=.89)$. El reactivo con la media más alta fue "Tengo amigos en mi salón de clases" $(\mathrm{M}=5.61, \mathrm{DE}$ $=1.04)$. Por el contrario, el ítem más bajo fue "Es difícil para mi maestro entender opiniones diferentes a la suya" $(\mathrm{M}=3.97, \mathrm{DE}=1.91)$.

Finalmente, el tercer componente nombrado "facilidad institucional" alcanzó un $4.90 \%$ de la varianza con cargas factoriales entre .38 y .63 con una confiabilidad de .75 con una media de $M=5.06$ $(D E=0.84)$. El ítem con la media más alta fue "Durante la clase mi maestro se esfuerza por asegurar que en mi salón de clases todos tengamos las mismas oportunidades" ( $M=5.45$, $D E=1.13)$, mientras que el reactivo con la media más baja fue "Puedo expresar mis creencias y formas de pensar sin temor a que me rechace mi maestro" ( $\mathrm{M}=4.67, \mathrm{DE}=1.69)($ Ver Tabla 3$)$.

Tabla 3. Estadísticos descriptivos y cargas factoriales de la escala de percepción de inclusión docente.

\begin{tabular}{cccccc}
\hline & & & \multicolumn{2}{c}{ Cargas factoriales } \\
\hline & Reactivos & $\boldsymbol{M}$ & $\boldsymbol{D E}$ & $\mathbf{1}$ & $\mathbf{2}$ \\
\hline 1. & Casi siempre mi maestro se muestra positivo & 5.10 & 1.32 & 0.588 &
\end{tabular}




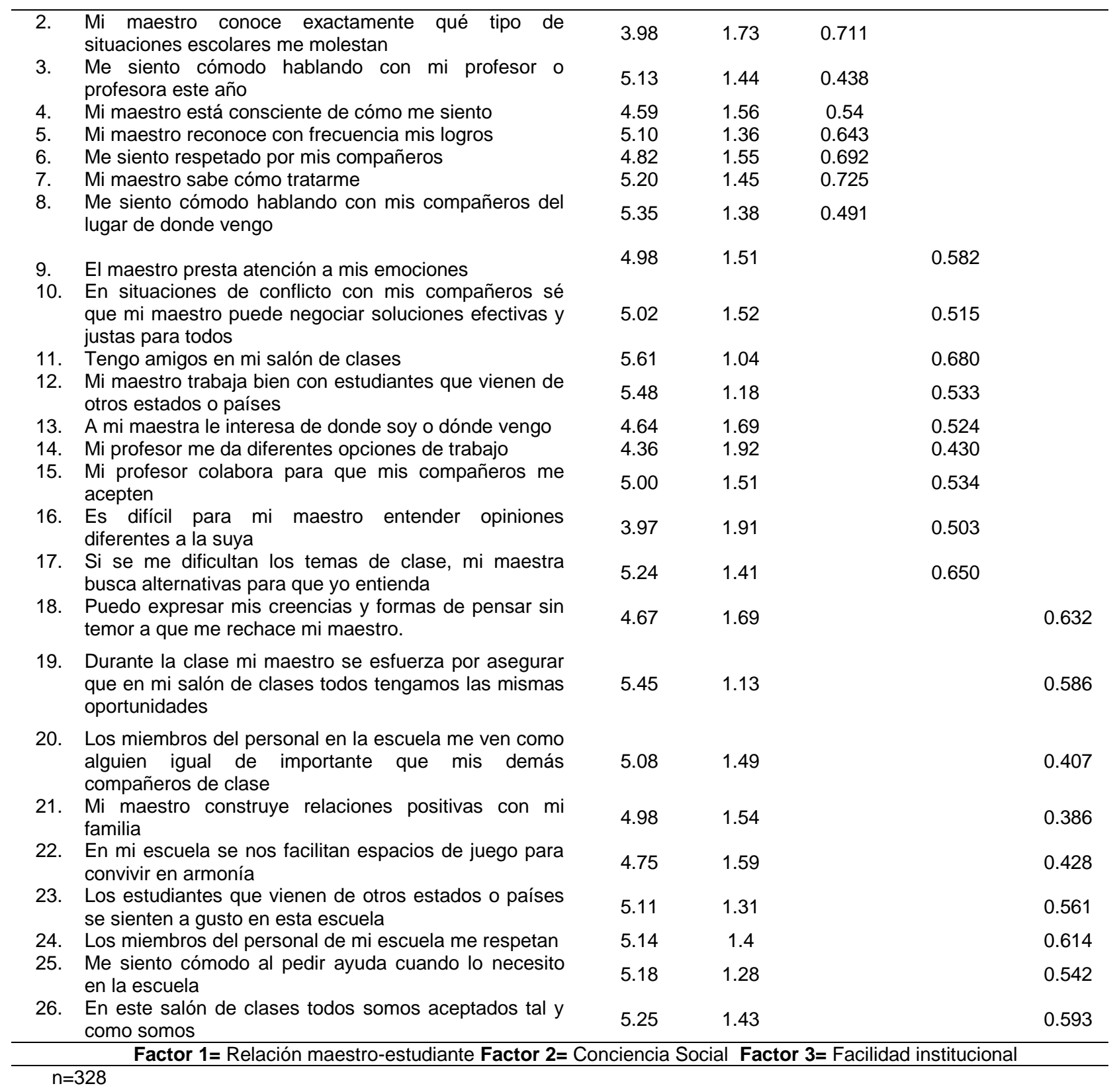

Con base a los análisis de correlación de Pearson, se exhiben las correlaciones entre las variables que conforman a los factores de segundo orden, donde es posible aprecia que todas las correlaciones resultaron altas, positivas y significativas entre los factores de la escala de percepción de inclusión docente $(p<.01)$ (Ver Tabla 4).

Tabla 4. Correlaciones entre los factores de percepción al docente

\begin{tabular}{lccc}
\hline & $\begin{array}{c}\text { Relación maestro- } \\
\text { estudiante }\end{array}$ & Conciencia social & Facilidad institucional \\
\hline Relación maestro-estudiante & 1 & 1 & \\
Conciencia social & $.74^{* \star}$ & $.70^{* \star}$ & 1 \\
\hline Facilidad institucional & $.69^{* \star}$ & & \\
\hline
\end{tabular}

Nota. ${ }^{*} p<.05 .{ }^{*} p<.01$ 


\section{Análisis factorial confirmatorio: (modelo estructural de la escala de percepción de inclusión docente.)}

En la Figura 1, se muestra el modelo estructural de la escala de percepción de inclusión docente. En el modelo se muestran los factores de primer orden (maestro-estudiante, conciencia social y facilidad institucional) que predicen al factor de segundo orden nombrado "percepción de inclusión docente". Para los tres factores de primer orden se computaron tres parcelas que sirvieron como indicadores para cada uno de los constructos.

El factor de segundo orden emerge de manera coherente a partir de las interrelaciones altas y significativas entre sus indicadores de primer orden. Las cargas factoriales indican validez convergente de constructo para los factores. Los indicadores de bondad de ajuste práctica resultaron adecuados $\left(x^{2}=26.71,[19 \mathrm{gl}], \mathrm{p}=.111\right)$ al igual que los índices de bondad de ajuste estadística (BNNFI=.98, BNNFI=.99, CFI= .99) y el $\mathrm{RMSEA}=.03$. Los resultados señalan que el modelo teórico se ajusta adecuadamente a los datos empíricos.

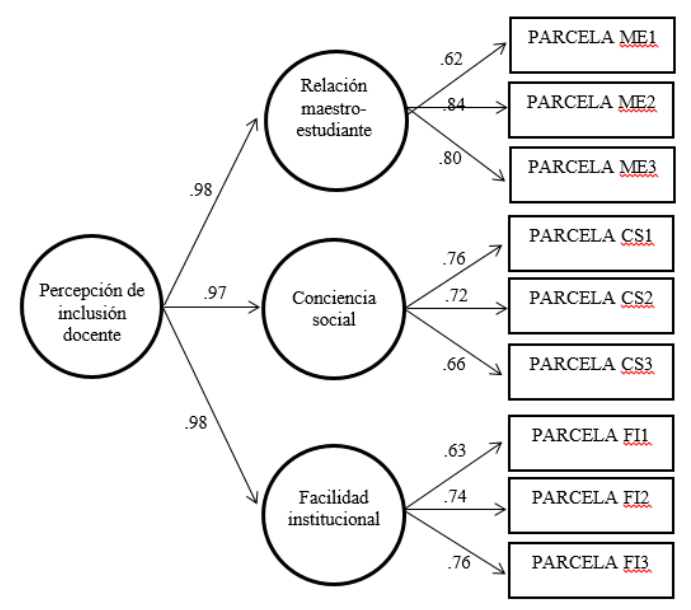

Figura 1. Modelo de covarianzas de la escala de percepción de inclusión docente. $X^{2}=26.71$, (19 gl), $\mathrm{p}=.111 ; \mathrm{BNNFI}=.98, \mathrm{BNNFI}=.99, \mathrm{CFI}=.99, \mathrm{RMSEA}=.03$

\section{Discusión}

Los resultados de la presente investigación demuestran que la escala de Percepción de Inclusión Docente (PID), presentó adecuadas propiedades psicométricas de validez y confiabilidad y una estructura factorial coherente entre los factores de la escala. Los análisis apoyan el modelo teórico hipotetizado de origen, presentando medidas adecuadas de homogeneidad en los ítems propuestos y una adecuada consistencia interna de la escala (alfa=.90), lo que indica que el cuestionario (PID) resulta satisfactorio para medir la Percepción de Inclusión Docente y este puede ser aplicado tanto a niños regulares y como a niños migrantes de segundo y tercer ciclo (primaria alta).

La niñez migrante interna o externa que llega al estado de Sonora, puede atravesar diferentes barreras para su adaptación al nuevo entorno educativo. Los grupos minoritarios suelen pasar por desapercibidos en el aula; no solo ante sus maestros, también para los directivos o sus compañeros de clase. Numerosos estudios demuestran que la ausencia de inclusión puede dañar el desarrollo emocional del estudiante e inclusive su desempeño académico, lo que puede desencadenar diversas problemáticas que se reflejan posteriormente en la sociedad a corto o largo plazo. En este sentido, diversas investigaciones ponen de manifiesto la importancia de las competencias socioemocionales de docentes y estudiantes para la calidad educativa (Vázquez, 2018)

Los registros y la trayectoria migratoria de las poblaciones migrantes, son importantes para conocer cifras reales y poder atender las numerosas necesidades que caracterizan a este importante sector de la población. Sin embargo, no hay datos sistematizados reales que permitan localizar y categorizar a las poblaciones migrantes en México. La información recopilada a través del Programa Binacional de Educación Migrante (PROBEM), nos dio una base para la ubicación de los 
planteles educativos que albergan estudiantes migrantes internacionales pero los datos no coincidían siempre con los datos reales, y la información es útil solo para la ubicación del estudiante que proviene de Estados Unidos. Es necesario identificar con mayor eficacia a los estudiantes migrantes internacionales, nacionales o de origen indígena para que no pasen como "invisibles" y puedan considerarse como estudiantes con vulnerabilidad para la institución, en especial para el personal docente y de esa forma, se atiendan las desigualdades con el respeto a su bagaje cultural, fortalecimiento de su idioma nativo y del contexto escolar; así como atención a los procesos emocionales. Con lo anterior, se puede evitar reprobación o deserción, y así contribuir a formar ciudadanos para la sociedad actual y globalizada.

Este estudio no está exento de limitaciones. En primer lugar, se trabajó a través de un auto informe, lo que podría estar sujeto a un cierto grado de subjetividad. Si bien, el auto informe puede resultar más factible para la medición, algunas sugerencias sería llevar a cabo estudios longitudinales que analicen la estabilidad de la escala y la estabilidad del modelo estructural propuesto en este estudio y observar si existe un cambio de comportamiento en la percepción de los mismos sujetos de acuerdo al tiempo que llevan en el nuevo plantel educativo que los alberga para lo que se requeriría un estudio longitudinal en futuras investigaciones. El acceso a las instituciones fue restringido y algunas escuelas de los registros oficiales no estaban en la dirección proporcionada.

Por otro lado, algunos directivos se mostraban desconfiados y se rehusaban a participar. Nos encontramos con planteles que restringen la entrada a investigadores, como los planteles localizados en los ejidos o algunas escuelas públicas. Lo anterior alargó el tiempo planeado para recopilación de información. Pese a las limitaciones, el presente trabajo presenta implicaciones importantes, ya que los resultados pueden ser tomados en cuenta para el desarrollo de futuros trabajos de investigación en este campo de estudio y para el desarrollo de legislaciones y políticas públicas en materia de inclusión que beneficien a los niños migrantes, ya sean de origen indígena, migrantes nacionales o extranjeros.

Con este trabajo, se pretende demostrar la importancia que tiene la inclusión de estudiantes migrantes, conocer cómo se siente la niñez migrante en su nuevo entorno escolar respecto a sus docentes, institución y compañeros de clase. Adicionalmente, la escala (PID) permite detectar las necesidades educativas especiales que tienen los niños migrantes, y en este sentido, la institución educativa que los alberga puede tomar acción para mejorar las condiciones de educabilidad, de igual forma, el maestro a cargo puede adaptar sus estrategias para el apoyo del niño en el aula. Conociendo las necesidades específicas de los estudiantes migrantes, se pueden abrir programas puente o bien, protocolos para la inserción del niño en situación migratoria al sistema educativo mexicano. Se concluye que para que el proceso de enseñanza-aprendizaje se pueda lograr con éxito, se deben tomar en consideración las fuerzas contextuales (raza, idioma, contexto familiar, economía, etc.) para la adaptación a las necesidades educativas de cada estudiante atendiendo la parte emocional y académica de cada sujeto, lo que pudiera ser considerada en la agenda política como el derecho a la educación (Constitución Política de los Estados Unidos Mexicanos, 1917, art. 3).

\section{Referencias}

Amat, O. (1998). Aprender a enseñar. Una visión práctica de la formación deformadores. $4^{\mathrm{a}}$ edición. Barcelona: Gestión 2000.

Arnaiz, P. (2003). Educación Inclusiva: Una escuela para todos. Málaga: Aljibe.

Bisquerra, R. (2001). Educación emocional y bienestar. Barcelona: Wolters Kluwer.

Chiner, E., \& Cardona, M. C. (2013). Inclusive education in Spain: how do skills, resources, and supports affect regular education teachers' perceptions of inclusion? International ournal of inclusive education, 17(5), 526-541.

Constitución Política de los Estados Unidos Mexicanos (1917). Diario oficial de la Federación. Recuperado de: http://www.diputados.gob.mx.

Corral, V. Frias, M. y González, D. (2001). Análisis Cuantitativo de Variables Latentes (Col. Tetos Académicos, Vol.13) México: UNISON.

Cruz, R. (2007) Educación inclusiva, profesores y estudiantes con discapacidad: una mirada desde lo relacional Sinéctica. Puebla, México. Recuperado de:

https://sinectica.iteso.mx/index.php/SINECTICA/article/view/964 
Validación de la escala de percepción de inclusión docente de la niñez migrante (PID)

D'Haem, J., \& Griswold, P. (2017). Teacher educators' and student teachers' beliefs about preparation for working with families including those from diverse socioeconomic and cultural backgrounds. Education and Urban Society, 49(1), 81-109.

Domović, V., Vidović Vlasta, V., \& Bouillet, D. (2017). Student teachers' beliefs about the teacher's role in inclusive education. European Journal of Special Needs Education, 32(2), 175-190.

Espinoza, E. (2006). Impacto del maltrato en el rendimiento académico. Electronic Journal of Research in Educational Psychology, 4(2), 221-238.

Field, A. (2013). Discovering statistics using IBM SPSS statistics. Sage.

Forlin, C., \& Chambers, D. (2011). Teacher preparation for inclusive education: Increasing knowledge but raising concerns. Asia-Pacific Journal of teacher education, 39(1), 17-32.

Gao, W., \& Mager, G. (2011). Enhancing preservice teachers' sense of efficacy and attitudes toward school diversity through preparation: A case of one US inclusive teacher education program. International Journal of Special Education, 26(2), 92-107.

Hsieh, W. Y, Hsieh, C. M., Ostrosky, M., \& McCollum, J. (2012). Taiwanese first-grade teachers' perceptions of inclusive education. International Journal of Inclusive Education, 16(1), 71-88.

Hu, L. T., \& Bentler, P. M. (1995). Measuring model fit. Structural equation modeling: Concepts, issues and applications. Thousand Oaks: Sage.

INEE (2017). Directrices para mejorar la atención educativa de niñas, niños y adolescentes indígenas. México: Estimaciones del CONAPO, con base en la Encuesta Intercensal 2015.

INEGI (2004). El rezago educativo en la población mexicana. Impreso en México. Recuperado de: http://internet.contenidos.inegi.org.mx/contenidos/productos/prod serv/contenidos/espanol/bvineg i/productos/historicos/2104/702825497538/702825497538 1.pdf

INEGI (2018). Características educativas de la Población. Recuperado de: https://www.inegi.org.mx/datos/

Li, X., Zou, H., \& Wang, L. (2009). A comparative study on school adjustment between migrant children in public schools and those in migrant worker children's schools. Chinese Journal of Special Education, 9(81), 6.

Moiica, A. (2017), Adolescentes y ióvenes en la migración de retorno a Michoacán, en El impacto sociocultural del fenómeno migratorio en Michoacán, Michoacán de Ocampo,

Monsen, J. J., Ewing, D. L., y Kwoka, M. (2014). Teachers' attitudes towards inclusion, perceived adequacy of support and classroom learning environment. Learning Environments Research, 17(1), 113-126.

Monzalvo, A. (en prensa). Competencia Socioemocional Docente para la inclusión y motivación escolar de niños migrantes en Hermosillo, México y área capital de Finlandia. (Tesis de Doctorado) División de Ciencias Sociales Posgrado Integral en Ciencias Sociales. Universidad de Sonora. México.

OCDE (2017). Employment Outlook 2016. Recuperado de https://bit.ly/3efnT0w

Restrepo, L. F., \& González, J. (2007). From pearson to Spearman. Revista Colombiana de Ciencias Pecuarias, 20(2), 183-192.

Rowan, L., Kline, J., \& Mayer, D. (2017). Early career teachers' perceptions of their preparedness to teach "diverse learners": Insights from an Australian research project. Australian Journal of Teacher Education (Online), 42(10), 71-92.

Secretaría de Educación Pública (2006). Diario oficial. Tercera sección poder ejecutivo secretaria de educación pública. Recuperado de: https://bit.ly/2W3oD2B

Tangen, D., \& Beutel, D. (2017). Pre-service teachers' perceptions of self as inclusive educators. International journal of inclusive education, 21(1), 63-72.

Tejada, A. J. R., Del Pino, R. M. C., Tatar, M., \& Sayáns, P. J. (2012). "Spanish as a foreign lanquage" teachers' profiles: inclusive beliefs, teachers' perceptions of student outcomes in the TCLA program, burnout, and experience. European journal of psychology of education, 27(3), 285-298.

Unesco (2015). La Educación para Todos, 2000-2015: logros y desafíos. París: Unesco.

Young, K., McNamara, P. M., \& Coughlan, B. (2017). Authentic inclusion-utopian thinking?-Irish post-primary teachers' perspectives of inclusive education. Teaching and Teacher Education, 68, $1-11$. 